\title{
Intraoperative Cytology Staining Technique
}

\subsection{Modified Field Stain}

Intraoperative consultation plays a crucial role in neurosurgical practice. It is routinely done by frozen sections and crush cytology smears. Crush cytology smears are helpful in setups where frozen section facility is not available. We have included intraoperative cytology pictures in this book.

Many staining techniques have been described in the literature like rapid hematoxylin-eosin stain, May-Grunwald-Geimsa (MGG), quick PAP, QuickDiff stain. We describe for the first time a modification of the Field stain for interpretation of intraoperative cytology, thereby reducing the turnaround time of staining to about 2 minutes, which is considerably shorter. Field's stain is a version of Romanowasky stain used for rapid processing of the specimens. It is commonly used for staining thick smears in detection of malarial parasites. The modification of the field stain is rapid, reliable, and provides high-quality cytologic detail for intraoperative crush smear interpretation. It is an easy and simple technique. The smears are fixed in $70 \%$ ethanol, following which only two reagents are required for staining. The intensity of staining can be adjusted according to the preference of the signing out pathologist. It is possible to stain the tissue darker or fainter within a matter of seconds. This staining method has yielded an excellent correlation between cytologic and histopathologic diagnosis.
The representative tumor tissue measuring $1 \mathrm{~mm} \times 1 \mathrm{~mm}$ is placed on the edge of a clean glass slide with the help of a blunt forceps and scalpel. Another clean glass slide is placed over the tissue, and with optimal pressure the tissue is crushed to make a thin smear.

\section{Procedure of Modified Field Stain for Squash Cytology}

1. Squash smears are air-dried.

2. Fixed with $70 \%$ ethanol.

3. Field A-12 to 15 dips (10 seconds).

4. Water wash.

5. Field $\mathrm{B}-8$ to 10 dips ( 8 seconds).

6. Water wash.

7. Field $\mathrm{A}-12$ to 15 dips (10 seconds).

8. Wash and view under microscope (can be coverslipped later).

\section{Advantages of the Modified Field Staining Method}

1. Staining takes less than 2 minutes.

2. The procedure has only two reagents and three steps.

3. Economical, highly reproducible.

4. Good correlation with histological diagnosis.

5. Fungi can also be detected.

6. The same slide may be used for Ziehl-Neelsen stain after complete destaining if a granulomatous lesion is suspected on squash. 